Research Article,

\title{
Enhanced Wound Healing With Caloric Restriction, Refeeding, And Intermittent Fasting: Dietary Strategies In Support Of Bioproliferative Injection Therapies
}

\author{
Inklebarger $\mathbf{J}^{1}$ \\ The London College of Osteopathic Medicine, 8-10 Boston Place, London NW1 6QH, UK
}

\begin{abstract}
:
Caloric restriction followed by re-feeding (CRRF), Intermittent fasting (IF) and ketogenic diets (KD) are known to be supportive of wound healing in animal models. Evidence also suggests that injections of bioproliferative solutions (prolotherapy) in combination with functional range of motion exercises also promote tissue regeneration via wound healing mechanisms. Intermittent fasting and Ketogenic diets also favor wound healing, and may therefore synergistically support the regenerative effects of prolotherapy. A systemic review of Pubmed, Google Scholar, and other databases was conducted. Though no published sources directly linking these types of diets to an enhanced bio-proliferative injection response were identified, several studies did demonstrate that bio-regenerative responses of CRRF diets and prolotherapy share a common wound-healing pathway. However, further research would be required to confirm and quantify if KD, CRRF, or IF diets are complimentary to prolotherapy.
\end{abstract}

Keywords: Prolotherapy, Bioproliferative Injections, Regenerative Medicine, Intermittent Fasting, Caloric Restiction, Diet, Ketogenic, Ketosis, Wound Healing

\section{Introduction:}

Prolotherapy, also known as regenerative injection therapy,

Is a portmanteau for bio-proliferation injection therapy? Also known as bongling or non-surgical joint reconstruction, it is a regenerative injection technique, in which small quantities of an inflammatory-inducing solution are introduced to the site of painful and degenerated tendon-bone insertions (enthesis), joints, ligaments, and adjacent joint spaces. Usually, several sessions are required to optimally stimulate regenerative processes. 1, 2, 3 Systemic database reviews, meta analysis, and rct trials have provided evolving evidence that injection of glucose-based solutions and other substances such as phenol-glyceringlucose into soft tissues and joints, stimulate and propagate regeneration effects via the woundhealing cascade. ${ }^{4,5,6,7}$ There is also evidence base that caloric restriction followed by re-feeding (crrf), intermittent fasting (if) and ketogenic diets (kd) also augments wound healing. ${ }^{8}$ As prolotherapy and diet-selective utilization of ketone energy substrates both appear to enhance tissue regeneration via wound healing pathways, it is proposed that these diets may also support the bioproliferative effects of prolotherapy. Recovery from tissue injury occurs via initial and delayed inflammatory phases. The initial influx of granulocytes attract macrophages, which in turn secrete debriding enzymes, chemotactants, and polypeptide growth factors. These secretions activate fibroblastic collagen deposition and wound repair. This tissue remodeling continues for many months after the initial injury, and in animal models, maximum wound strength is achieved circa one year after injury. So, wound healing progresses through staged granulocytosis, collagen deposition, scar formation/reformation, and movement enhanced functional remodeling over several months. Wolf's law regarding bone remodeling and the Davis' soft tissue law of 1867 are both in accordance with the 'specific adaption to imposed demands principle. Functional 
movements also optimize tendon-ligament and volume remodeling over several months by inhibiting scar tissue formation, preserving optimal range of motion, and promoting collagen re-arrangement into more functional orderly arrays. ${ }^{9,10,11,12}$

\section{Discussion:}

On a search of the literature, the first historical references to ketogenic diets date back to 1924 investigations into the management of epilepsy. ${ }^{13}$ in more recent times $\mathrm{KD}$ has been regarded as a broad-spectrum therapy for medically intractable epilepsy. ${ }^{14}$ As the brain requires a steady supply of glucose (120 mg per day), serum insulin decreases and the liver begins production of ketone bodies from corporeal stores or ingested fat, with the ketones used by the brain as an alternative source of fuel. ${ }^{15}$ It follows that peripheral nerves may also benefit from using ketones as a primary fuel source, which has a potential for supporting bioproliferative tissue regeneration objectives. Glucose solution injections may also play a role in the pain neuromodulation. Though controversial, some studies have proposed that dextrose-based subcutaneous peri-peripheral nerve injections and caudal epidurals may inhibit neurogenic inflammation by modulation of trpv 1 receptors. ${ }^{16}$, 17, 18 Ketotic substrate energy metabolism related to cancer, was researched by Dr. Otto Warburg, a german-jewish physiologist, medical doctor and friend of albert Einstein in the 1930's Germany. Though his notorious associations with Adolf Hitler and the nazi regime grossly limited international acceptance of his ideas, he was nevertheless nominated for the Nobel Prize 47 times. Warburg's original work with sea-urchin respiration, determined that cancer cells selectively ferment sugar even in the presence of oxygen (anaerobic glycolysis), and die without it while healthy cells did not. This original research landed him Nobel laureate in 1931. ${ }^{19}$ Though some studies have reported that restricted energynutrient intake is associated with age-related impairment of wound healing, $20,21,22$ caloric restriction-re-feeding and ketogenic diets enhance wound healing recovery in animal models, and also support metabolic, endocrine and neurological functions.

However, caloric restriction (cr) followed by ad libitum re-feeding prior to wounding (RF), was noted to enhance wound healing in both rats and primates. ${ }^{23}$ Selective carbohydrate restrictive fatbased ketogenic diets which favor ketone ( $\beta$ hydroxybutyrate $(\beta h b)$ ) energy metabolism also appear to augment wound healing. ${ }^{8}$ There appear to be several ketogenic diet variations, such as cyclical eating of carbohydrates and/or additional amounts of protein. However the standard ketogenic diet typically contains $70 \%$ fat, $20 \%$ protein, and $10 \%$ carbohydrate $\left(<50 \mathrm{~g}\right.$ per day) ${ }^{24}$ As in the stages of wound healing and remodeling, prolotherapy appears to support tissue regeneration via transient stimulation of the inflammation cascade. In contrast, systemic and protracted inflammation associated with chronic hyperglycemic states places wound in a perpetual state of inflammation, which inhibits healing. ${ }^{25}$ Protein and fat rich animal foods are generally age rich and subject to further age formation through malliard cooking reactions. ${ }^{26}$ However, only the low molecular weight ages are associated with diet, with vegetarian diets counter-intuitively inducing higher overall age concentrations in comparison to non-vegetarians. The evidence base implies that hyperglycemia rather than dietary age intake is responsible for chronic inflammatory states. ${ }^{27}$ Activation of the age receptors (rage) favors cytokine production (tissue necrosis factor b), and inhibition of metalloproteinase. 28 dietary carbohydrate restrictions also favors the decreased formation of pro-inflammatory age related glycation end products (ages). Diabetes type $2(\mathrm{dm})$ case series have demonstrated the effectiveness of therapeutic fasting to reverse insulin resistance, with resultant cessation of insulin therapy, accompanied by reduction in body fat, weight circumference and glycated haemoglobin level. ${ }^{29}$

Though not directly related, improved glycemic contol and associated weight loss may also support a response to prolotherapy treatments. There are over 100 known physiologic factors contributory to wound healing deficiencies in diabetes, such as impairment of growth factor production, ${ }^{30}$ macrophage function, [mayurama $\mathrm{k}$ et al 2007], collagen accumulation, epidermal barrier integrity, an granulation tissue generation $^{32}$. Therefore, improving patient serum glucose levels is probably also an imperative for optimization of the prolotherapy response. In more general laboratory rat and mice if and crrf 
studies, a broad spectrum of health indices has been noted, notably on disease processes (diabetes, cardiovascular disease, cancers, alzheimer's, Parkinson's, seizure disorders, CVA recovery), with improved functional outcomes. Body fat loss, enhancement of adaptive cellular stress response signaling pathways, enhanced mitochondrial health, DNA repair and autophagy, stem cell-based regeneration; prolonged metabolic improvements have been also reported. Intermittent fasting diets implemented in rat studies pre-spinal cervical cord-lesioning, have demonstrated a neuroprotective effect, with increased plasticity, and promotion of motor recovery. ${ }^{33}$ Placing time restrictions on feeding have been shown to have broad systemic effects and trigger similar biological pathways as caloric restriction. ${ }^{34}$ The utility of alternate day fasting as also been explored. ${ }^{35}$ Though there is less is known about the role of exercise on wound healing, ${ }^{23}$ caloric restrictions do not appear to impact an individuals' ability to engage in moderate exercise. A twelve-week combined cr and exercise trial amongst subjects with obesity reported equal attendance to a supervised exercise programme (40 min of $75 \%$ max heart rate on three days per week) on both restricted and nonrestricted feeding days. ${ }^{36}$ Similarly, carter and colleagues in 2016, 34 reported a comparable increase in daily average step count in the both if and cr groups and hill et al, reported comparable and good adherence to a moderate intensity walking programme (five 20-50 min sessions of brisk walking $60 \%-70 \%$ max heart rate per week) amongst dieters undertaking if/cr also ${ }^{37}$ Fasting combined with exercise also shows an enhanced release of growth hormone. There is evidence that myocellular GH signaling is stimulated after exercise and fasting in terms of increased stat5 phosphorylation and/or igf-i gene expression. This suggests that exercise with brief, well-defined GH peaks leads to distinct stat5 phosphorylation and subsequent igf-i gene expression, whereas fasting induces more sporadic GH bursts and less distinct but more persistent activation of the GH signal. ${ }^{38}$ However the type of exercise (running vs. Weight training) may also be important to consider in goals of tissue regeneration. It has been demonstrated that genetically mutated and extremely obese $\mathrm{db} / \mathrm{db}$ mice (leptin-compromised) mice become hyperglycemic after forced treadmill exercise, which is thought to be in response to excessive corticosterone and catecholamine production. Excessive norepinephrine secretion during acute exercise has also been reported in hypertensive and non-hypertensive type 2 diabetic patients, and poor metabolic control is associated with post exercise hyperglycemia and hyperinsulinemia in patients with type 2 diabetes. 39,40

Furthermore weight loss via cr is accompanied by a significant decrease in lean body mass $(\mathrm{lbm})^{41}$

A loss of muscle mass is also associated with frailty and increased mortality at advanced ages, and is a challenge for successful aging ${ }^{42}$ However a meta-analysis reviewing factors of exercise, training, aging, sarcopenia, muscle mass, strength training, caloric restriction diets concluded that crrt (cr with resistance training) is able to prevent almost $100 \%$ of cr-induced muscle loss, while resulting in $\mathrm{fbm}$ and bm reductions that do not significantly differ from cr. ${ }^{43}$ Effects of cr on agerelated impairments in wound healing in rodents have been variable, ${ }^{20,21,22}$ In general, restricted animals have not demonstrated improvements in wound healing and in some cases even appear to be more impaired than age-matched, ad libitumfed, senescent counterparts ${ }^{21}$ [reiser et al., 1995]. However, at least one report has suggested that if restricted animals are re-fed ad libitum before wounding, healing rates improve markedly and are superior to those in non restricted controls ${ }^{22}$ [reed et al., 1996]. Even less is known about the role of exercise on wound healing. ${ }^{23}$ However, functional movements due promote healing of more functional scar tissue. ${ }^{11}$ During fasting and after exercise, skeletal muscle efficiently switches from carbohydrate to lipid as the main energy source to preserve glycogen stores and blood glucose levels for glucose-dependent tissues. Skeletal muscle cells sense this limitation in glucose availability and transform this information into transcriptional and metabolic adaptations. ${ }^{44}$ Animal-derived foods that are high in fat and protein are generally age related glycation endproduct (age) rich, and are prone to further age formation during cooking. However, only low molecular weight ages are absorbed through diet, and vegetarians have been found to have higher concentrations of overall ages compared to nonvegetarians.Some authors consider the relatively high-fructose content of vegetarian diets is 
responsible for higher ages than meat eaters. An alternative explanation is that the relatively poor taurine status of vegetarians and vegans. 45 Furthermore, glycation cross-line malliard reactions in meat dishes are significantly reduced by acidic ingredients such as lemon juice \& vinegar, moist heat-low temperature cooking, shortened cooking times, and age inhibitory compounds such as aminoguanidine. ${ }^{26}$ It is therefore unclear whether dietary ages contribute to disease and aging. However, decreasing glucose dietary uptake and controlling the blood glucose level reduce carbonyl stress involved in the formation of endogenous ages. Thus there is mitochondrial oxidative stress relief through selective reliance of ketogenic diets, ${ }^{46}$ which have a potential to limit the formation of endogenous ages, ${ }^{47}$ and meta-analysis has demonstrated agerelated delayed wound closure. ${ }^{48}$

By implication crrf, if, and KD all a potential to reduce oxidative stress on tissue and support the effects of bi-proliferative injections.

\section{Discussion:}

Both wound healing and regenerative injection responses occur through the conflicting activity of macrophage enzymatic intercellular matrix degradation versus continued granulocytic fibroblastic deposition of new collagen. It is the new collagen that gives strength to the healing wound and prolotherapy-treated tissues, with denovo collagen synthesis and deposition necessary for repair and regeneration in the late inflammatory phase. ${ }^{49}$ Prolotherapy and diets favoring ketogenesis both appear to augment regeneration through the wound-healing cascade. Dextrose prolotherapy may also promote tissue repair that is required for healing chronic wounds and ameliorating the associated pain. ${ }^{50}$ There also appears to be some benefit with short term if, crrf, kds in support of prolotherapy treatment. However caution is required, as even short term carbohydrate restriction has some potential complications such as transient hunger, headache, constipation, low mood fatigue, irritability and loss of concentration with these symptoms particularly affecting the obese. ${ }^{51}$ The most common if-crrf early-onset complication appears to be dehydration, especially in patients who started dietary ketosis with initial fasting. Gastrointestinal (gi) disturbances, such as nausea/vomiting, diarrhea, and constipation, also appear to be frequently noted, sometimes associated with gastritis and fat intolerance. Other early-onset complications, in order of frequency, were hypertriglyceridemia, transient hyperuricemia, hypercholesterolemia, susceptibility infectious diseases, symptomatic hypoglycemia, hypoproteinemia, hypomagnesemia, repetitive hyponatremia, low concentrations of high-density lipoprotein, lipoid pneumonia due to aspiration, hepatitis, acute pancreatitis, and later complications of persistent metabolic acidosis. ${ }^{52}$ Advice for these diets therefore warrants risk-benefit awareness, particular in subjects with multiple co-morbidities, endocine-compromise, the elderly, immunocompromised, and during pregnancy \& breastfeeding. Following known wound healing time frames and other augmenting wound healing factors, compliance with graded functional range of motion exercises, activity modifications, and avoidance of overtraining may also be complimentary to regenerative injections both during and up to one year after prolotherapy, with if/crrf/kd potentially augmenting functional tissue remodeling. Protracted wound remodeling may also indicate that these diets would support an optimal bio-proliferative tissue response if followed both during and several months after prolotherapy sessions. However, the time window remains unknown. Further research will be required, both to verify and more fully understand the potential role these diets may play in prolotherapy, and recognize complications associated with these diets.

Funding: no external funding sources Competing interests: none

\section{References:}

[1.] Linetsky fs, Rafael m, saberski 1. Pain management with regenerative injection therapy (rit) in: weiner rs, editor. Pain management. Boca Raton: crc press; 2002. Pp. 381-402. [Google scholar]

[2.] Adams e. Bibliography: prolotherapy for musculoskeletal pain. Boston, ma: veterans; 0000. [google scholar]

[3.] Goswami a. Prolotherapy. J pain palliat care pharmacother. 2012; 26:376- 
8. [Pubmed] [google scholar]

[4.] Banks a. A rationale for prolotherapy. J orthop med. 1991; 13(3): 54-59

[5.] Ongley mj, Klein rg, dorman ta, eek BC, Hubert $\mathrm{lj}$. A new approach to the treatment of chronic low back pain. Lancet. 1987 July 18; 2:143-146. [Pubmed]

[6.] Rabago d., slattengren a. \& zgierska a. Prolotherapy in primary care practice. Prim. Care. 37(1), 65-80 (2010). [Pmc free article] [Pubmed] [Google scholar]

[7.] Sit rws, chung vch, reeves kd, rabago d, chan kkw, chan dcc, ho rst, won sys, hypertonic dextrose injections (prolotherapy) in the treatment of symptomatic knee osteoarthritis: a systematic review and meta-analysism 2016, sci rep, 2016;6:252_doi: 10.1038/srep25247

[8.] Hunt nd, li gd, min $z$, levette a, chachich me, spangler el, allard js, hyun d-h, ingram $\mathrm{dk}$, de cabo $\mathrm{r}$, effect of calorie restriction and refeeding on skin wound healing in the rat, age (dordr)_2012 dec; 34(6): 14531458.

[9.] Nutt, john joseph (1913). Diseases and deformities of the foot, e.b. Treat \& company.

[10.] Frost hm, Wolff's law and bone's structural adaptations to mechanical usage: an overview for clinicians. Angle orthod. 1994; 64:175188. [Pubmed] [Google scholar]

[11.] Frost $h$, new targets for fascial, ligament and tendon research: a perspective from the Utah paradigm of skeletal physiology" $\mathrm{j}$ musculoskel neuron interact 2003; 3(3):201-209

[12.] Ehrilich hp, control of wound healing from connective tissue aspect [article in german], chirug, 1995; 66(3):165-73

[13.] Wheless jw. History of the ketogenic diet. Epilepsia. 2008; 49(suppl 8):35. [Pubmed] [Google scholar]

[14.] Gano lb, patel m, rho Jm. Ketogenic diets, mitochondria, and neurological diseases. $\mathrm{J}$ lipid res. 2014; 55(11):2211-28. [Pmc free article] [Pubmed] [Google scholar]

[15.] Paoli a, rubii a, volek js, grimaldi ka, beyond weight loss: a review of the therapeutic uses of very-low-carbohydrate (ketogenic) diets, eur $\mathrm{j}$ clin nutr, 2013:67(8):789

[16.] Geppetti, et al. Neurogenic inflammation. Boca Raton: edited crc press; 1996. Chapter 5, summary; p.53-63.

[17.] Levi-montalcini r, et al. Developmental neurobiology and the natural history of nerve growth factor, ann. Rev. Neurosc. 1982; 5:341-62.

[18.] Maniquis-smigel $\mathrm{l}$, reeves $\mathrm{kd}$, rosen $\mathrm{hj}$, lyftogt j, graham-coleman $d$, cheng al, rabago $\mathrm{d}$, short term analgesic effects of $5 \%$ dextrose epidural injections for chronic low back pain: a randomized controlled trial, anesth pain med. $2017 \mathrm{feb}$; 7(1): e42550.

[19.] Otto am, Warburg effect(s) — a biographical sketch of otto Warburg and his impacts on tumor metabolism, cancer metab, 2016; 4:5

[20.] Harrison, d.e.; archer, j.r. Biomarkers of aging: tissue markers. Future research needs strategies, directions and priorities. Exp. Gerontol, 1988; 23:309-21

[21.] Reiser, k.; McGee, c; rucker, r.; McDonald, r. Effects of aging and caloric restriction on extracellular matrix biosynthesis in a model of injury and repair in rats. $J$. Gerontol. Biol. Sci, 1995; 50a:b40-b47

[22.] Reed, m.j.; penn, p.e.; li, y.; birnbaum, r.; vernon, r.b.; johnson, t.s.; pendergrass, w.r.; sage, e.h.; abrass, j.b.; wolf, n.s. Enhanced cell proliferation and biosynthesis mediate improved wound repair in refed, caloric-restricted mice. Mech. Ageing dev. 1996; 89:21-43

[23.] Roth gs, kowatch ma, hengemihle dk, Ingram el, spangler Lk, johnson mal, effect of age and caloric restriction on cutaneous wound closure in rats and monkeys, the journals of gerontology: series a, volume 52a, issue 2, march 1997, pages b98-b102.

[24.] Shilpa j, mohan v, ketogenic diets: boon or bane, Indian $\mathrm{j}$ of med res, 2018; 148(2): 


\section{1-53}

[25.] Salazar JJ, ennis wj, koh tf, diabetes medications: impact on inflammation and wound healing, $\mathrm{j}$ of diabetes complications, 2016; 30(4):746-52

[26.] Uribarri, Jaime; et al, "advanced glycation end products in foods and a practical guide to their reduction in the diet", journal of the American dietetic association. 110, 2010 ;(6): 911916. Doi:10.1016/j.jada.2010.03.018. Pmc 3 704564. Pmid 20497781

[27.] Poulsen, malene w.; hedegaard, rikke v.; Andersen, Jeanette m.; de courten, barbora; bügel, Susanne; Nielsen, john; skibsted, leif h.; dragsted, Lars o. (October 2013). "Advanced glycation endproducts in food and their effects on health". Food and chemical toxicology. 60: 10-37.

[28.] Yan HD, li xz, xie jm, li m (2007). "Effects of advanced glycation end products on renal fibrosis and oxidative stress in cultured nrk-49f cells". Chin. Med. J. 120 (9): 787-93.

[29.] Fumil s, elmasry, ramos $m$, fung j, therapeutic use of intermittent fasting for people with type 2 diabetes as an alternative to insulin, bmj case rep. 2018, doi: 10.1136/bcr-2017-221854

[30.] Galkowska h., wojewodzka u., olszewski w.l. Chemokines, cytokines, and growth factors in keratinocytes and dermal endothelial cells in the margin of chronic diabetic foot ulcers. Wound repair regen. 2006; 14:558565. [Pubmed] [Google scholar]

[31.] Galiano r.d., et al. Topical vascular endothelial growth factor accelerates diabetic wound healing through increased angiogenesis and by mobilizing and recruiting bone marrow-derived cells. . Am. J. Pathol. 2004; 164:19351947. [Pmc free article] [Pubmed] [Google scholar]

[32.] Falanga v. Wound healing and its impairment in the diabetic foot. Lancet. 2005; 366:17361743. [Pubmed] [Google scholar]
[33.] Jeong $\mathrm{m}$, plumet $\mathrm{w}$, streiliger $\mathrm{f}$, lee jht, plemal jr, park s, lam ck, tetzleff w, intermittent fasting improves functional recovery after rat thoracic contusion spinal cord injury, j neurotrauma, 2011, 28(3): 479-492.

[34.] Anton s, leeuwenburgh c. Fasting or caloric restriction for healthy aging. Exp gerontol. 2013; 48(10):1003-5. [Pmc free article] [Pubmed] [Google scholar]

[35.] Varady ka, Bhutani s, klempel mc, et al. Alternate day fasting for weight loss in normal weight and overweight subjects: a randomized controlled trial. Nutr j. 2013;12(1):146. [Pmc free article] [Pubmed] [Google scholar]

[36.] Bhutani s., klempel M.C., kroeger c.m., trepanowski j.f., varady k.a. Alternate day fasting and endurance exercise combine to reduce body weight and favorably alter plasma lipids in obese humans. Obesity. 2013; 21:1370-1379. Doi: $\quad$ 10.1002/oby.20353. [Pubmed] [Crossref] [Google scholar]

[37.] Hill j.o., schlundt d.g., sbrocco t., sharp t., pope-cordle j., stetson b., kaler m., heim c. Evaluation of an alternating-calorie diet with and without exercise in the treatment of obesity. Am. J. Clin. Nutr. 1989; 50:248-254. [Pubmed] [Google scholar]

[38.] Vendelbo $\mathrm{mh}$, jorgenson jo, pederson sb, gormsen lc, lund s, schmitz o, jessen $n$, moller $\mathrm{n}$, exercise and fasting activate growth hormone-dependent myocellular signal transducer and activator of transcription-5b phosphorylation and insulin-like growth factor-i messenger ribonucleic acid expression in humans. $\mathbf{J}$ clin endocrinol metab. 2010; 95(9):e64-8. Doi: 10.1210/jc.2010-0689. Epub 2010 Jun 9.

[39.] Kjaer $m$, hollenbeck cb, frey-hewitt b, galbo h, haskell $\mathrm{w}$, reaven $\mathrm{gm}$. Glucoregulation and hormonal responses to maximal exercise in non-insulin-dependent diabetes. J appl physiol. 1990; 68(5):20672074. [Pubmed] [Google scholar] 
[40.] Hubinger a, franzen a, gries fa. Hormonal and metabolic response to physical exercise in hyperinsulinemic and nonhyperinsulinemic type 2 diabetics. Diabetes res. 1987;4(2):57-61. [Pubmed] [Google scholar]

[41.] Weinheimer E.M., sands 1.p., Campbell w.w. A systematic review of the separate and combined effects of energy restriction and exercise on fat-free mass in middleaged and older adults: implications for sarcopenic obesity. Nutr. Rev. 2010; 68:375-388. Doi: 10.1111/j.17534887.2010.00298.x. [Pubmed]

[Crossref] [Google scholar]

[42.] Fielding R.A., vellas b., Evans w.j., bhasin s., Morley j.e., Newman a.b., abellan van Kan g., andrieu s., bauer j., breuille d., et al. Sarcopenia: an undiagnosed condition in older adults. Current consensus definition: prevalence, etiology, and consequences. International working group on sarcopenia. J. Am. Med. Dir. Assoc. 2011; 12:249-256. Doi: 10.1016/j.jamda.2011.01.003. [Pmc free article] [Pubmed] [Crossref] [Google scholar]

[43.] Sardeli av, komatsu TR, mori ma, gaspan $\mathrm{AF}$, chacon-mikahil mpt, resistance training prevents muscle loss induced by caloric restriction in obese elderly individuals: a systematic review and meta-analysis, nutrients. 2018 apr; 10(4): 423. Published online $2018 \quad$ mar 29. Doi: $10.3390 /$ nu 10040423

[44.] Canto c, jiang lq, deshmukh as, et al. Interdependence of ampk and sirt1 for metabolic adaptation to fasting and exercise in skeletal muscle. Cell metab. 2010;11(3):213-9. [Pmc free article] [Pubmed] [Google scholar]

[45.] Mccarthy mf, he low-age content of low-fat vegan diets could benefit diabetics - though concurrent taurine supplementation may be needed to minimize endogenous age production, med hypotheses.2005; 64(2):394-8.

[46.] Guibaud a, niquet-leridon c, boulanager e, tessier f, how can diet affect the accumulation of advanced glycation endproducts in the human body?, foods, 2016; 5(4): 84

[47.] Greco t, glenn tc, hovda da, prins $\mathrm{ml}$, ketogenic diet decreases oxidative stress and improves mitochondrial respiratory complex activity, $\mathrm{j}$ cereb blood flow metab, 2016;

36(9): 1603-1613.

[48.] Putte lv, schrijver SD, moorgat $p$, the effects of advanced glycation end products (ages) on dermal wound healing and scar formation: a systematic review, scars burn heal, 2016;

Doi: $10.1177 / 2059513116676828$

[49.] Clark ra, regulation of fibroplasia in cutaneous wound repair, j cell biol, 1985; 101:2330-34

[50.] Siadat ah, isseroff RR, prolotherapy: potential for the treatment of chronic wounds? Published online: 3 apr 2019 https://doi.org/10.1089/wound.2018.0866

[51.] Lawlor t, wells dg. Metabolic hazards of fasting. American journal of clinical nutrition. 1969; 22(8):1142-

9. [Pubmed] [Google scholar]

[52.] Kang hc, chung de, kim dw, kim hd, earlyand late-onset complications of the ketogenic diet for intractable epilepsy, epilepsia, 2004;45(9):1116-23. Carter s., Clifton p.m., keogh j.b. The effects of intermittent compared to continuous energy restriction on glycaemic control in type 2 diabetes; a pragmatic pilot trial. Diabetes res. Clin. Pract. 2016; 122:106-112.Doi:

10.1016/j.diabres.2016.10.010. [Pubmed] [Crossref] [Google scholar] 